\title{
Analysis of transcriptomic changes in bovine endometrial stromal cells treated with lipopolysaccharide
}

\section{Xuefen Ding}

Henan Agricultural University

Haimiao Lv

Henan Agricultural University

\section{Lixin Deng}

Henan Agricultural University

\section{Wenju Hu}

Henan Radio and Television University

\section{Zhan Peng}

Henan Agricultural University

Chenbo Yan

Henan Agricultural University

\section{Dexin Yang}

Henan Agricultural University

\section{Chao Tong}

Henan Agricultural University

Xinzhuang wang ( $\square$ happywang169@163.com )

Henan Agricultural University https://orcid.org/0000-0002-2410-9831

\section{Research article}

Keywords: Whole-genome sequencing, Endometritis, Bovine endometria stromal cells, Lipopolysaccharide

Posted Date: November 6th, 2019

DOl: https://doi.org/10.21203/rs.2.16904/v1

License: (c) (1) This work is licensed under a Creative Commons Attribution 4.0 International License.

Read Full License 


\section{Abstract}

Background: Endometritis adversely affects the ability of cattle to reproduce, and significantly reduces milk production. Consequently, it has great influence on the economic value of dairy cows. The endometrium is mainly composed of epithelial and stromal cells and they produce the first immune response to invading pathogens. Epithelial cells are the first cellular barrier through which bacteria enter the uterine endometrium. However, most of the epithelial cells are disrupted and stromal cells are exposed to an inflammatory environment when endometritis occurs, especially postpartum. Inflammation in endometrial stromal cells is thus activated, immune-related cytokines and signaling pathways are activated. This indicates that endometritis is becoming more and more serious. However, inflammatory response in bovine endometrial stromal cells is yet to be studied in detail. Understanding the genomewide characterization of the bovine endometritis will be beneficial for prevention and treatment of endometritis. In this study, whole-transcriptomic gene changes in bovine stromal cells treated with LPS were compared with those treated with PBS (control group) and were analyzed by RNA sequencing (RNAseq). Results: Compared with the control group, a total of 366 differentially expressed genes (DEGs) were identified in LPS-induced group (234 upregulated and 132 downregulated genes), with an adjusted P value $\mathbb{X} 0.05$ by DESeq. Gene ontology (GO) enrichment analysis revealed DEGs were most enriched in lymphocyte activation, interleukin-1 receptor binding, regulation of cell activation, and lymphocyteactivated interleukin-12 production. Kyoto Encyclopedia of Genes and Genomes (KEGG) pathway analysis revealed DEGs were most enriched in TNF signaling pathway, Toll-like receptor signaling pathway, cytokine-cytokine receptor interaction, nucleotide-binding oligomerization domain-like (NOD-like) receptor signaling pathway, NF-kB signaling pathway, and chemokine signaling pathway. Conclusion: Our results proved and expanded findings of previous studies on bovine endometritis. These results will be useful to propose new therapeutic targets and eliminate or reduce inflammation by comprehending molecular mechanisms and authenticating unique genes related to endometritis.

\section{Background}

Endometritis is caused by a highly inflammatory environment created by local microbe-associated molecular patterns (MAMPs), resulting in subclinical or clinical disease [1,2]. Endometritis severely affects the ability of cattle to reproduce, compromises animal welfare, and significantly reduces milk production. As a consequence, it has great influence on the economic value of dairy cows [3, 4]. Bacterial infection is the most prevalent element of bovine endometritis, especially, Escherichia coli. Once endometrium is infected with $E$. coli, it promotes invasion of other bacteria into the uterus after few days of calving [5]. Lipopolysaccharide (LPS) is a vital constituent of the outer membrane of gram-negative bacteria which can mimic characteristics of an actual gram-negative bacterial infection [6]. Previous studies have reported expression changes in peroxisome proliferator-activated receptors (PPARs) after intrauterine injection of E. coli suspension in vivo and in endometrial explants with exposure to LPS in vitro. Their findings indicate that LPS exposure can mimic inflammation in vitro, which is useful for 
understanding intracellular mechanisms during endometritis, and specific PPAR ligands could potentially be used to cure endometritis [7].

The endometrium is mainly composed of epithelial cells and stromal cells and they produce the first immune response to the invading pathogens [8]. Epithelial cells are the first cellular barrier through which bacteria enter the uterine endometrium and they play a significant role in bacterial detection in the uterine lumen [9]. However, most epithelial cells are disrupted and stromal cells are exposed to an inflammatory environment when endometritis occurs, especially postpartum. Consequently, inflammation is activated in endometrial stromal cell [10], and immune-related cytokines and signaling pathways are activated. This also indicates that endometritis is getting worse. In addition, previous reports pointed out that stromal cells play a significant role in initiating immunological response and they are even more important than epithelial cells for endometritis, as stromal cells are more accessible to the vascular system and mononuclear cells, thus, the effect of stromal cytokines and chemokines is more significant $[10,11]$. Moreover, stromal cells outnumber epithelial cells in the endometrium and secrete soluble growth factors as much as epithelial cells in order to increase immunological function of uterus endometrium [9, 12]. However, the inflammatory response of bovine endometrial stromal cells remains to be studied in detail.

Genome-wide characterization is crucial for understanding development and pathophysiology of bovine endometritis, which will be beneficial for prevention and treatment of endometritis. High-throughput sequencing technologies provide a platform for insights into genomic characterization. Specifically, highthroughput mRNA sequencing (RNA-seq) enables concurrent transcript discovery and abundance estimation [13]. Previous studies on RNA-seq analysis in mixed bovine epithelial and stromal endometrial cells treated with LPS showed 20 differentially-expressed miRNAs and 108 DEGS, as well as enriched 118 GO and 66 KEGG pathways connected to them [14]. In addition, a previous study on RNA-seq analysis of change in miRNA and mRNA expression in 15 cows at 7 and 21 days postpartum showed 4197 DEGs in healthy cows, while only 31 genes were differentially expressed in cows with cytological endometritis from proinflammatory to hyperplasia stages [15]. Other studies have been performed on RNA-seq analysis of effects of LPS on whole transcriptome of bovine endometrial epithelial cells with focus on genes involved in embryo-maternal interactions. They identified $2 \varangle 035$ and $2 \varangle 073$ DEGs in control cells and cells treated with 2 and $8 \mu \mathrm{g} / \mathrm{mL}$ LPS for $24 \mathrm{~h}$, respectively [16]. However, comprehensive transcriptomic analysis of response of bovine endometrial stromal cells to LPS by RNA-seq remains to be elucidated.

In this study, we observed whole transcriptomic changes in primary bovine endometrial stromal cells treated with LPS using RNA-seq. This study could act as a supplement to former published information on bovine endometritis. In addition, our research has presented basic features of the transcriptional architecture of stromal cells treated with LPS. These data may promote better understanding of gene expression in the stromal cells treated with LPS.

\section{Results}




\section{Quality control of the RNA sequence}

In this study, we established six RNA libraries for control group and LPS-induced group. After filtering the low-quality reads, the average number of clean reads was 42,381,010 (92.67\%) and 44,167,270 (92.77\%) for the control group and LPS-induced group, respectively (Table 1). The clean reads were used for the following analyses and most of them (>93.99\%) were mapped to the Bos taurus genome (Table 2).

\section{Identification of the source of variance in the expressed transcripts by principal component analysis (PCA)}

The purpose of PCA analysis is to account for the origin of variance in our data. We performed PCA analysis with two elements: PC1 and PC2. PC1 was 55\% and PC2 was 42\% (Additional file 1: Figure S1). PCA analysis can cluster similar samples together, the closer the distance, the higher the similarity between samples. This indicated that these data could be used further.

\section{Differential gene expression}

There were a total of 366 DEGs (composed of 234 upregulated and 132 downregulated DEGs) were identified in LPS-induced group compared to control group. Some of these genes are related to inflammation, such as inflammatory cytokines including $I L 1 A$, IL2RB, and IL6, increased 3.16ه20.53, 15.88 fold, respectively. Chemokines including CCL2, CCL5, CXCL8, and CXCL2, increased 16.91, 10.48, $3.39,2.14$ fold respectively. Antimicrobial factor DEFB 10 increased 2.79 fold (Figure 1 and Additional file 2: Table S1).

\section{The heatmap of DEGs}

Heatmap analysis of DEGs identified genes of expression levels with high correlation among samples. Some of these genes may be involved in biological processes, metabolic processes, or signaling pathways (Figure 2).

\section{GO enrichment of DEGs}

GO enrichment was used to characterize DEGs. DEGs were enriched in immunity-related GO terms, such as leucocyte-mediated immunity, intracellular signal transduction, IL-1 receptor binding, $I L-1 \beta$ secretion, and response to cytokines. There were $638 \mathrm{GO}$ terms, including 574 biological process (BP), 21 cellular component (CC), and 43 molecular function (MF). GO enrichment results for all DEGs are illustrated (Additional file 3, Table S2), and the top $20 \mathrm{GO}$ terms with the most significant enrichment were selected (Figure 3).

\section{KEGG enrichment analysis}

The purpose of KEGG enrichment analysis is to appraise pathways that are vital in inflammation in endometrial stromal cells. The results of the analysis showed that inflammation pathways in endometrial stromal cells are mainly centered on TNF signaling pathway, complement and coagulation cascades, 
cytokine-cytokine receptor interaction, IL-17 signaling pathway, chemokine signaling pathway, nucleotidebinding oligomerization domain-like (NOD-like) receptor signaling pathway, NF-kB signaling pathway, and so on. KEGG enrichment results of DEGs are illustrated (Additional file 4, Table S3). The top 20 KEGG terms with the most significant enrichment were selected (Figure 4).

\section{Confirmation of DEGs by quantitative reverse-transcription PCR (qRT-PCR)}

We selected six DEGs which included five upregulated genes, NFKBIZ, NFKBIA, CCL2, C3, and STEAP4 and one downregulated gene, $\angle 1 C A M$, and confirmed them by real time qRT-PCR (as shown in Figure 5). The expression level of the six DEGs were found to be essentially in agreement with RNA-seq results.

\section{Discussion}

In this study, we described whole transcriptomic genes changes in bovine endometrial stromal cells treated with LPS compared with control group by RNA-seq. Stromal cells are exposed to an inflammatory environment when epithelial cells are disrupted (Figure 6), inflammation on endometrial stromal cells is activated (Figure 7 ) $[1,10]$. Results indicate that more than $97 \%$ of clean tags of RNA-seq results mapped to the genome and PCA was low, which proved our data could be used for functional analyses.

LPS binds to lipoprotein binding protein (LBP), which presents it to CD14 receptor. This induces CD14 to present LPS-LBP complex to MD-2 (myeloid differentiation factor 2) $[17,18]$. This, in turn, promotes dimerization of toll-like receptor 4 (TLR4)/MD-2, activating two downstream signaling pathways including MyD88-dependent pathway (MyD88-) and TRIF-dependent pathway. The former triggers NF-KB and mitogen-activated protein kinase (MAP kinase) signaling and induces inflammatory cytokines, and the latter causes the induction of type I interferons (IFN) through interferon regulatory factor 3 (IRF3) activation and inflammatory cytokines through NF-KB activation [17]. Our data presents evidence for the activation of the MyD88-independent pathway as LPS upregulated inflammatory cytokines, such as IL6, IL 1A, CXCL8 and activated NF-KB pathway (Additional file 2: Table S1 and Additional file 4: Table S3). Bovine endometrial epithelial and stromal cells express the TLR4/CD14/MD2 receptor complex. We found increased expression levels of CD14 but constant levels of TLR4 or MyD88 in our study in which bovine stromal cells were treated for $12 \mathrm{~h}$ with $0.5 \mu \mathrm{g} / \mathrm{mL}$ LPS (Additional file 2: Table S1), consistent with previous studies that exposed mixed bovine epithelial and stromal endometrial cells to $100 \mathrm{ng} / \mathrm{ml}$ LPS for $6 \mathrm{~h}$ [19]. In addition, LPS upregulated TLR4 only in bovine endometrial epithelial cells which were treated with $1 \mu \mathrm{g} / \mathrm{mL}$ LPS for $6 \mathrm{~h}$ and 12h, and the expression was greatest at $12 \mathrm{~h}$ [20].

After the membrane surface receptor is recognized, intracellular inflammation is recognized and intracellular signaling cascades are activated by LPS, which is mediated by innate pattern recognition receptors (PRR) including TLR, retinoic acid-inducible gene--like (RIG-I-like) receptors, NOD-like receptors, and C-type lectin receptors [21]. These lead to the expression of inflammatory mediators and contribute to the clearance of pathogens [22]. Our data further confirm these findings because LPS triggers NOD-like receptor signaling pathway, TLR signaling pathway, and C-type lectin receptor signaling pathway in bovine endometrial stromal cells (Additional file 4, Table S3). In addition, our RNA-seq results also 
demonstrate the upregulation of expression of inflammatory and chemotactic cytokines related genes, such as IL6, IL 1A, IL2RB, CCL2, CCL5, CXCL5, CXCL8, CXCL2, CX3CL 1, and CCL20 (Additional file 2: Table $\mathrm{S} 1)$. This is also consistent with previous studies that mRNA of proinflammatory cytokines IL $1 A$, IL1B, $I L 6, T N F$, and expression of chemokines IL 8 and $C X C L 5$ increased in endometrial epithelial cells during the estrous cycle and subclinical or clinical endometritis [23,24]. The expression of these cytokines was also upregulated in only epithelial cells, only stromal cells, and mixed epithelial and stromal cells treated with LPS in vitro $[12,19,25]$. On the other hand, activation of PRR also triggers many signal transduction pathways through one or more of the IRF family of transcription factors, leading to the expression of IFNs [26]. LPS upregulated the expression IFN-stimulated genes (ISG) including RSAD2, MX2, OAS1Y, ISG15, and $B S T 2$ in mixed epithelial and stromal cells [19]. In the present study, our results show that LPS upregulated the expression of ISG15 and IRF1, 3.71 fold and 2.36 fold, respectively (Additional file 2: Table S1).

Local immune responses are activated which result in expression of proinflammatory cytokines when the uterus is exposed to bacteria. This is followed by production of antimicrobial peptides (AMP) and acute phase proteins by epithelial and innate immune cells [10]. Many of AMP genes are expressed in bovine endometrium, including lingual antimicrobial peptide $(L A P)$, tracheal antimicrobial peptide (TAP), and some $\beta$-defensins $[10,27,28]$. Defensins are small cationic polypeptides, which have antimicrobial and immunomodulatory properties and are secreted by many cell types [29]. Some studies have identified an increase in expression levels of TAP, LAP, DEFB1, and DEFB5 in endometrium of cows with serious inflammation or following LPS treatment in vitro $[20,25,30]$. Other studies showed LAP, TAP, neutrophil $\beta$ defensins (BNBD4, DEFB5) were all upregulated in bovine epithelial cells but not stromal cells with LPS [8, $10]$. In the present study, we found DEFB10 was upregulated 2.79 fold in bovine stromal cells with LPS (Additional file 2: Table S1)..

Cascade reaction of complement signals also plays a key role in immune defense, and complement activation lead to opsonization of pathogens and their removal by phagocytes as well as cell lysis [31]. Some studies pointed out that complement factors $C 2$ and $C F B$ increased in primary cultures of mixed bovine epithelial and stromal endometrial cells with LPS [19]. Our results agreed with previous studies and identified that the expression of other complement genes were upregulated, such as C3,C1S, C1R, $C F 1$, and C4A (Additional file 2: Table S1). LPS can change expression level of many genes involved in cell adhesion, such as VCAM1, SELP, CADM2, HEPACAM, and SDK2 (Additional file 2: Table S1).

Leucocytes attach to cell adhesion molecules (CAM) on endothelial cells and are involved in inflammation and immune function [32]. Each CAM has an inherent effect on immune response process, such as vascular cell adhesion molecule-1 (VCAM-1), which promotes firm binding of T cells and induces trans-migration [33] In addition, there were reports in the 80s that CAMs may play a role in inherited inflammatory disease, which is characterized by deficient leucocyte CAMs [34].

This study also proves that that LPS can alter other gene expression levels in stromal cells, such as hyaluronan synthase 2 (HAS2), which showed 3.36 fold increase in expression. It belongs to the hyaluronan (HA) family, holds extracellular matrix (ECM) polysaccharides and has multiple functions, 
such as tissue repair and promoting the expression of inflammation-related genes including $T N F, I L 12$, $I L 1 B$, and MMPs [35]. Six-transmembrane epithelial antigen of prostate 4 (STEAP4), which showed 81.57 fold increase in expression, is an anti-inflammatory protein induced by inflammatory signals such as TNFa and IL6 [36-38]. Notably, mice with STEAP4-knockout are susceptible to insulin resistance, hyperglycemia, and inflammation [39].

In brief, we identified a total of 366 DEGs in bovine endometrial stromal cells in LPS-induced group as compared to the control group. We analyzed the interactions between LPS and stromal cells including interaction of LPS with surface receptors of stromal cells and intracellular signaling cascades activated by PRRs. These indicate that stromal cells play a key role in bovine endometrium reaction to foreign intruders by initiating or inhibiting biochemical and molecular signals. In addition, our results identified many important immune-related genes and signaling pathways, especially, IL6, IL 1A, IL2RB, CCL2, CCL5, CXCL5, CXCL8, CXCL2, and CX3CL 1, and TNF signaling pathway, NF-KB signaling pathway. Our results also expound the cellular processes in the endometrium and may serve as a diagnostic norm of defining and detecting endometritis, which may assist in development of more targeted and effective vaccines and drugs for the prevention and treatment of bovine endometritis. Furthermore, our analysis paves the way for the future elucidation of molecular mechanism of microbial invasion and host cell response. In our next study, we will continue to analyze and validate immune-related proteins and KEGG signaling pathways by western blot and compare genes and proteins in stromal cells treated with LPS in vitro with stromal cells from bovine endometritis with subclinical or clinical disease. These can better elucidate characteristics of bovine endometritis.

\section{Conclusions}

In summary, we identified a total of 366 DEGs, comprising of 243 upregulated and 132 downregulated DEGs in bovine endometrial stromal cells in LPS-induced group compared to control group. These DEGs were validated by $638 \mathrm{GO}$ terms and 31 by KEGG enrichment. Our results showed that most DEGs were enriched in immunity-related signaling pathways such as TNF signaling pathway, complement and coagulation cascades, cytokine-cytokine receptor interaction, NOD-like receptor signaling pathway, NF-kB signaling pathway, and chemokine signaling pathway. These results proved and expanded findings of previous studies on bovine endometritis and will be useful to propose new therapeutic targets and minimize or eliminate inflammation by uncovering the underlying molecular mechanisms and authenticating unique genes related to endometritis.

\section{Methods}

\section{Bovine endometrial stromal cell isolation and primary culture}

Whole stromal cells were isolated from bovine endometrium as described before with some changes [40, 41]. All experimental procedures were approved by Institutional Animal Use Committee of Henan 
Agricultural University (approval number. 2005-0026) and Beijing Association for Science and Technology (approval SYXK [Beijing]

2007-0023). Bovine uterus, stored on ice, was transported to the laboratory from a local abattoir. The endometrium was cut into strips and placed in serum-free DMEM/F12 (Gibco, Grand Island, NY, USA) containing $50 \mathrm{IU} / \mathrm{mL}$ penicillin, $50 \mu \mathrm{g} / \mathrm{mL}$ streptomycin, and $2.5 \mu \mathrm{g} / \mathrm{mL}$ amphotericin B. Then, endometrial strips were cut into about $1 \mathrm{~mm}^{3}$ fragments and placed in phosphate-buffered saline (PBS). They were then digested with $30 \mathrm{~mL}$ sterile digestive solution, composed of $60 \mathrm{mg}$ trypsin III, $60 \mathrm{mg}$ collagenase II, and $12 \mu \mathrm{L}$ deoxyribonuclease I in $120 \mathrm{~mL}$ PBS at $37^{\circ} \mathrm{C}$ for about $1 \mathrm{~h}$. The cell suspension was filtered with a $40 \mu \mathrm{m}$ mesh and the filtrate was resuspended with $2 \mathrm{~mL}$ DMEM/F-12 containing 10\% fetal bovine serum (FBS, Gibco, Grand Island, NY, USA). After washing the cells five times with DMEM/F12 containing $10 \%$ FBS, the filtrate was centrifuged at $100 \mathrm{rpm}$ for $10 \mathrm{~min}$ at room temperature (RT). The cells were resuspended in DMEM/F-12 containing 15\% FBS, $100 \mathrm{U} / \mathrm{mL}$ penicillin, $100 \mathrm{U} / \mathrm{mL}$ streptomycin, and $100 \mathrm{U} / \mathrm{mL}$ amphotericin B. Cells were seeded in $25 \mathrm{~cm}^{2}$ culture flask at a density of $2 \times 10^{5}$ cells $/ \mathrm{mL}$, and were incubated at $37^{\circ} \mathrm{C}$ with $5 \% \mathrm{CO}_{2}$. To obtain stromal cells and remove epithelial cells populations, the cell suspension was removed $18 \mathrm{~h}$ after plating, which allowed selective attachment of stromal cells. The medium was changed every two days. Cells were passaged with $0.25 \%$ trypsin-EDTA until the cells reached $\sim 80 \%$ confluence. Cell morphology was recorded and photographed under an inverted microscope. Stromal cells exhibited flat, fibroblast-like morphology. Endometrial stromal cells were estimated by immunostaining for vimentin (stromal cells marker) [42]. Stromal cells expression was vimentin-positive and cytokeratin-18 negative. Cells morphology and the positive staining for vimentin confirmed that the cultured cells were indeed stromal cells.

\section{Cells treatment with LPS}

Stromal cells from passage 6 were seeded in $25 \mathrm{~cm}^{2}$ culture flask at a density of $2 \times 10^{5}$ cells $/ \mathrm{mL}$. The cells were incubated at $37^{\circ} \mathrm{C}$ with $5 \% \mathrm{CO}_{2}$. Once the cells reached $80 \%$ confluence, the medium was removed and cells were washed with PBS. We divided the cells into two groups of three duplicates each. DMEM/F12 containing 15\% FBS and PBS (control) was added to one group and DMEM/F12 containing $15 \% \mathrm{FBS}$ and $0.5 \mu \mathrm{g} / \mathrm{mL}$ ultrapure LPS was added to the other group and incubated for $12 \mathrm{~h}$ each

\section{RNA extraction, quantification, and qualification}

Total RNA from stromal cells was extracted by RNAiso Plus (Takara, Dalian, Liaoning, China). Concentration and quality of RNA were estimated with NanoDrop spectrophotometer (Thermo Scientific) and integrity of RNA was verified with $1 \%$ agarose gel.

\section{Library preparation and transcriptome sequencing}

Input material of $3 \mu \mathrm{g}$ RNA was used for RNA sample preparation. Sequencing libraries were generated using the TruSeq RNA Library Preparation Kit v2 (Illumina, San Diego, CA, USA). In summary, poly-A containing mRNA was enriched by oligo-dT magnetic beads and fragmentation was completed by 
divalent cations under elevated temperature in an Illumina proprietary fragmentation buffer. Random oligonucleotides and SuperScript II were used for synthesizing first strand cDNA. Second strand cDNA was synthesized. Remaining overhangs were converted into blunt ends by exonuclease/polymerase. Before hybridization, 3 ' ends of the DNA fragments was adenylated and Illumina paired-end (PE) adapter oligonucleotides were ligated. The amplified library fragments were enriched using Illumina PCR primer cocktail in a 15-cycle PCR reaction, then the library size was selected at 300-400 bp fragments. AMPure XP system was used to purify PCR products and Bioanalyzer 2100 system (Agilent, Santa, Clara, CA, USA)) was used to detect library size. Finally, library sequencing of PEs was accomplished with NextGeneration Sequencing (NGS) on Illumina HiSeq platform.

\section{Quality control}

The raw data of FASTQ was generated by the software of the sequencing platform, Illumina HiSeq. Raw data of each sample was counted separately, including sample names, percentage of ambiguous base, Q20, and Q30 sequencing data along with some connectors and low-quality reads. These sequences may cause interference to subsequent data analysis, so sequencing data need to be further filtered. The criteria for data filtering included: 1 ) Cutadapt is used to remove 3 ' end connectors, and the removed parts have at least 10 bp overlap with known connectors, allowing $20 \%$ base mismatch; 2 ) removal of reads with average mass fraction lower than Q20.

\section{Mapping reads to the reference genome}

The reference genome used was of Bos taurus. ARS-UCD1.2.dna.toplevel.fa. were downloaded from Ensembl genome browser 95 . The clean reads were mapped to the reference genome with HISA2 Qhttp://ccb.jhu.edu/software/hisat2/index.shtml ).

\section{Gene expression level quantification}

The reads of each gene was counted by HTSeq and normalized by fragments per kilobase of transcript sequence per millions base pairs sequ.enced (FPKM). FPKM was then calculated [13].

\section{Analysis of DEGs}

Genes that were differentially expressed in two groups (three biological replicates per group) were screened with DESeq. The screening conditions for differentially expressed genes were |log2FoldChange| $>1$, significance of false discovery rate $(F D R)<0.05$. FDR is the adjusted $P$-value.

\section{Gene ontology (GO) and Kyoto Encyclopedia of Genes and Genomes (KEGG) enrichment analysis of DEGs}

We performed GO enrichment analysis for function annotation and KEGG enrichment analysis for signaling pathway annotation on DEGs $[43,44]$. GO and KEGG enrichment analyses were completed by 
the Database for Annotation, Visualization, and Integrated Discovery (DAVID) version 6.7 with threshold of FDR $<0.05$ [45].

\section{QRT-PCR}

Six DEGs were selected for confirmation of RNA-seq results by real time qRT-PCR. Total RNA was extracted by RNAiso Plus and reverse-transcribed into cDNA by a cDNA synthesis kit (Takara, Dalian, Liaoning, China) according to the manufacturer's instructions. Primer sequences listed (Additional file 5: Table S4) were used. The reaction mix ( $20 \mu \mathrm{L})$ comprised of the following: SYBR enzyme, $10 \mu \mathrm{L}$; forward and reverse primers, $1 \mu \mathrm{L}$; cDNA template, $1 \mu \mathrm{L}$; and dd $\mathrm{H}_{2} \mathrm{O}, 7 \mu \mathrm{L}$. Reaction conditions included predegeneration at $95^{\circ} \mathrm{C}$ for $30 \mathrm{~s}$, followed by 40 cycles of degeneration at $95^{\circ} \mathrm{C}$ for $5 \mathrm{~s}$, and annealing at $60^{\circ} \mathrm{C}$ for $30 \mathrm{~s}$. Glyceraldehyde 3-phosphate-dehydrogenase (GAPDH) was used as internal reference gene. Each sample was run three times. The relative expression level of target mRNAs were computed using the $2^{-\Delta \Delta C t}$ method [46].

\section{List Of Abbreviations}

LPS: Lipopolysaccharide; DEGs: Differentially expressed genes; GO: Gene ontology; KEGG: Kyoto Encyclopedia of Genes and Genomes; PPARs peroxisome proliferator-activated receptors; RNA-Seq: Highthroughput mRNA sequencing; PCA: Principal component analysis; LBP: lipoprotein binding protein; PRRs: Innate pattern recognition receptors; AMP: Antimicrobial peptides; LAP: Lingual antimicrobial peptide; TAP: tracheal antimicrobial peptide; MD-2: Myeloid differentiation factor 2; FDR: False discovery rate; IRF3: interferon regulatory factor 3; MAP kinase: Mitogen-activated protein kinase; ISG: IFNstimulated genes; GAPDH: Glyceraldehyde 3-phosphate-dehydrogenase; STEAP4: Six-transmembrane epithelial antigen of prostate 4; CAM: Cell adhesion molecules; VCAM-1: Vascular cell adhesion molecule1; HA: hyaluronan; ECM: extracellular matrix; HAS2: hyaluronan synthase 2

\section{Declarations}

\section{Ethics approval and consent to participate}

All experimental procedures were approved by Institutional Animal Use Committee of Henan Agricultural University (approval number. 2005-0026) and Beijing Association for Science and Technology (approval SYXK [Beijing] 2007-0023).

\section{Consent for publication}

Not applicable.

\section{Availability of data and materials}

The sequence data of this study have been deposited into Sequence Read Archive (http://www.ncbi.nlm.nih.gov/sra, accession number PRJNA574911). The datasets supporting the 
conclusions of this article are included in this article and its supplementary information files.

\section{Competing interests}

The authors declare that they have no competing interests.

\section{Funding}

This work was supported by the National Key Research and Development Program (2018YFD0501803, 2018YFD0501800), The Programmes of the National Beef Cattle and Yak Industrial Technology System (CARS-37) and Project for the Training of Young Cadre Teachers in Henan Higher Education Institutions in 2016(2016GGJS-282).

\section{Authors' contributions}

$X W, C T$ and XD designed the study and revised manuscript. XD, HL, LD, WH, ZP, CY, and DY performed the study. XD, HL, LD, CT and XW analyzed the data. XD, HL and LD wrote the manuscript. All authors read and approved the final manuscript.

\section{Acknowledgements}

Not applicable.

\section{References}

1. Kelly P, Meade KG, O'Farrelly C. Non-canonical Inflammasome-Mediated IL-1 beta Production by Primary Endometrial Epithelial and Stromal Fibroblast Cells Is NLRP3 and Caspase-4 Dependent. Front Immunol. 2019;10:102.

https://doi.org/10.3389/fimmu.2019.00102.

2. Chapwanya A, Meade KG, Foley C, Narciandi F, Evans AC, Doherty ML, et al. The postpartum endometrial inflammatory response: a normal physiological event with potential implications for bovine fertility. Reprod Fertil Dev. 2012;24(8):1028-1039.

https://doi.org/10.1071/RD11153.

3. Ribeiro ES, Lima FS, Greco LF, Bisinotto RS, Monteiro AP, Favoreto M, et al. Prevalence of periparturient diseases and effects on fertility of seasonally calving grazing dairy cows supplemented with concentrates. J Dairy Sci. 2013;96(9):5682-5697.

https://doi.org/10.3168/jds.2012-6335.

4. Bromfield JJ, Santos JE, Block J, Williams RS, Sheldon IM. PHYSIOLOGY AND ENDOCRINOLOGY SYMPOSIUM: Uterine infection: linking infection and innate immunity with infertility in the high- 
producing dairy cow. J Anim Sci. 2015; 93(5):2021-2033.

https://doi.org/10.2527/jas.2014-8496.

5. Dohmen MJ, Joop K, Sturk A, Bols PE, Lohuis JA. Relationship between intra-uterine bacterial contamination, endotoxin levels and the development of endometritis in postpartum cows with dystocia or retained placenta. Theriogenology. 2000;54(7):1019-1032.

https://doi.org/10.1016/s0093-691x(00)00410-6.

6. Beutler B, Hoebe K, Du X, Ulevitch RJ. How we detect microbes and respond to them: the Toll-like receptors and their transducers. J Leukoc Biol. 2003;74(4):479-485.

https://doi.org/10.1189/jlb.0203082.

7. Socha BM, Lada P, Szczepanska AA, Lupicka M, Korzekwa AJ. The influence of experimentally induced endometritis on the PPAR expression profile in the bovine endometrium. Theriogenology. 2018;122:74-83.

https://doi.org/10.1016/j.theriogenology.2018.09.013.

8. Davies D, Meade KG, Herath S, Eckersall PD, Gonzalez D, White JO, Conlan RS, O'Farrelly C,8 Sheldon IM: Toll-like receptor and antimicrobial peptide expression in the bovine endometrium. Reprod Biol Endocrinol. 2008;6:53.

https://doi.org/10.1186/1477-7827-6-53.

9. Wira CR, Fahey JV, Sentman CL, Pioli PA, Shen L. Innate and adaptive immunity in female genital tract: cellular responses and interactions. Immunol Rev. 2005; 206:306-335.

https://doi.org/10.1111/j.0105-2896.2005.00287.x.

10. Chapwanya A, Meade KG, Doherty ML, Callanan JJ, Mee JF, O'Farrelly C. Histopathological and molecular evaluation of Holstein-Friesian cows postpartum: toward an improved understanding of uterine innate immunity. Theriogenology. 2009;71(9):1396-1407.

https://doi.org/10.1016/j.theriogenology.2009.01.006.

11. Wagner WC, Hansel W. Reproductive physiology of the post partum cow. I. Clinical and histological findings. J Reprod Fertil. 1969;18(3):493-500.

https://doi.org/10.1530/jrf.0.0180493.

12. Cronin JG, Turner ML, Goetze L, Bryant CE, Sheldon IM. Toll-like receptor 4 and MYD88-dependent signaling mechanisms of the innate immune system are essential for the response to 
lipopolysaccharide by epithelial and stromal cells of the bovine endometrium. Biol Reprod. 2012;86(2):51.

https://doi.org/10.1095/biolreprod.111.092718.

13. Trapnell C, Williams BA, Pertea G, Mortazavi A, Kwan G, van Baren MJ, et al. Transcript assembly and quantification by RNA-Seq reveals unannotated transcripts and isoform switching during cell differentiation. Nat Biotechnol. 2010;28(5):511-515.

https://doi.org/10.1038/nbt.1621.

14. Wang J, Yan X, Nesengani LT, Yang L, Lu W. Integrated analysis of miRNA and mRNA expression profiling in bovine endometrial cells in response to lipopolysaccharide-stimulation. Microb Pathog. 2018; 114:129-138.

https://doi.org/10.1016/j.micpath.2017;11.053.

15. Foley C, Chapwanya A, Callanan JJ, Whiston R, Miranda-CasoLuengo R, Lu J,et al. Integrated analysis of the local and systemic changes preceding the development of post-partum cytological endometritis. BMC genomics. 2015;16:811.

https://doi.org/10.1186/s12864-015-1967-5.

16. Guo $\mathrm{Y}$, van Schaik T, Jhamat N, Niazi A, Chanrot M, Charpigny G, et al. Differential gene expression in bovine endometrial epithelial cells after challenge with LPS; specific implications for genes involved in embryo maternal interactions. PloS one. 2019;14(9):e0222081.

https://doi.org/10.1371/journal.pone.0222081.

17. Kawai T, Akira S. The role of pattern-recognition receptors in innate immunity update on Toll-like receptors. Nat Immunol. 2010;11(5):373-384.

https://doi.org/10.1038/ni.1863.

18. Herath S, Lilly ST, Fischer DP, Williams EJ, Dobson H, Bryant CE, et al. Bacterial lipopolysaccharide induces an endocrine switch from prostaglandin F2alpha to prostaglandin E2 in bovine endometrium. Endocrinology. 2009;150(4):1912-1920.

https://doi.org/10.1210/en.2008-1379.

19. Oguejiofor CF, Cheng Z, Abudureyimu A, Fouladi-Nashta AA, Wathes DC. Global transcriptomic profiling of bovine endometrial immune response in vitro. I. Effect of lipopolysaccharide on innate immunity. Biol Reprod. 2015;93(4):100.

https://doi.org/10.1095/biolreprod.115.128868.

Page $13 / 26$ 
20. Fu Y, Liu B, Feng X, Liu Z, Liang D, Li F, et al. Lipopolysaccharide increases Toll-like receptor 4 and downstream Toll-like receptor signaling molecules expression in bovine endometrial epithelial cells. Vet Immunol Immunopathol. 2013;151(1-2):20-27.

https://doi.org/10.1016/j.vetimm.2012.09.039.

21. Kayagaki N, Wong MT, Stowe IB, Ramani SR, Gonzalez LC, Akashi-Takamura S, et al. Noncanonical inflammasome activation by intracellular LPS independent of TLR4. Science. 2013;341(6151):12461249.

https://doi.org/10.1126/science.1240248.

22. Takeuchi O, Akira S. Pattern recognition receptors and inflammation. Cell. 2010; 140(6):805-820.

https://doi.org/10.1016/j.cell.2010.01.022.

23. Herath S, Lilly ST, Santos NR, Gilbert RO, Goetze L, Bryant CE, et al. Expression of genes associated with immunity in the endometrium of cattle with disparate postpartum uterine disease and fertility. Reprod Biol Endocrinol. 2009;7:55.

https://doi.org/10.1186/1477-7827-7-55.

24. Fischer C, Drillich M, Odau S, Heuwieser W, Einspanier R, Gabler C. Selected pro-inflammatory factor transcripts in bovine endometrial epithelial cells are regulated during the oestrous cycle and elevated in case of subclinical or clinical endometritis. Reprod Fertil Dev. 2010;22(5):818-829.

https://doi.org/10.1071/RD09120.

25. Swangchan-Uthai T, Lavender CR, Cheng Z, Fouladi-Nashta AA, Wathes DC. Time course of defense mechanisms in bovine endometrium in response to lipopolysaccharide. Biol Reprod. 2012;87(6):135.

https://doi.org/10.1095/biolreprod.112.102376.

26. Yanai $\mathrm{H}$, Taniguchi T. Fine-tuning type I IFN signaling: A new chapter in the IFN saga. Cell Res. 2017;27(12):1407-1408.

https://doi.org/10.1038/cr.2017.118.

27. Cormican P, Meade KG, Cahalane S, Narciandi F, Chapwanya A, Lloyd AT, et al. Evolution, expression and effectiveness in a cluster of novel bovine beta-defensins. Immunogenetics. 2008;60(3-4):147156.

https://doi.org/10.1007/s00251-007-0269-8. 
28. Severa M, Coccia EM, Fitzgerald KA. Toll-like receptor-dependent and -independent viperin gene expression and counter-regulation by PRDI-binding factor-1/BLIMP1. J Biol Chem. 2006;281(36):26188-26195.

https://doi.org/10.1074/jbc.M604516200.

29. Ganz T. Defensins antimicrobial peptides of innate immunity. Nat Rev Immunol. 2003;3(9):710-720. https://doi.org/10.1038/nri1180.

30. Wathes DC, Cheng Z, Chowdhury W, Fenwick MA, Fitzpatrick R, Morris DG,et al. Negative energy balance alters global gene expression and immune responses in the uterus of postpartum dairy cows. Physiol Genomics. 2009;39(1):1-13.

https://doi.org/10.1152/physiolgenomics.00064.2009.

31. Sarma JV, Ward PA. The complement system. Cell Tissue Res. 2011;343(1):227-235.

https://doi.org/10.1007/s00441-010-1034-0

32. Kim SK, Moon WK, Park JY, Jung H. Inflammatory mimetic microfluidic chip by immobilization of cell adhesion molecules for T cell adhesion. Analyst. 2012; 137(17):4062-4068.

https://doi.org/10.1039/c2an35424a.

33. Ferran C, Peuchmaur M, Desruennes M, Ghoussoub JJ, Cabrol A, Brousse N, et al. Implications of de novo ELAM-1 and VCAM-1 expression in human cardiac allograft rejection. Transplantation. 1993;55(3):605-609.

https://doi.org/10.1097/00007890-199303000-00026.

34. Allen S, Moran N. Cell Adhesion Molecules: Therapeutic Targets for Inhibition of Inflammatory States. Semin Thromb Hemost. 2015;41(06):563-571.

https://doi.org/10.1055/s-0035-1556588

35. Petrey AC, de la Motte CA. Hyaluronan, a crucial regulator of inflammation. Front Immunol. 2014;5:101.

https://doi.org/10.3389/fimmu.2014.00101.

36. Gomes IM, Maia CJ, Santos CR. STEAP proteins: from structure to applications in cancer therapy. Mol Cancer Res. 2012;10(5):573-587.

https://doi.org/10.1158/1541-7786.MCR-11-0281. 
37. Korkmaz CG, Korkmaz KS, Kurys P, Elbi C, Wang L, Klokk TI, et al: Molecular cloning and characterization of STAMP2, an androgen-regulated six transmembrane protein that is overexpressed in prostate cancer. Oncogene. 2005;24(31):4934-4945.

https://doi.org/10.1038/sj.onc.1208677.

38. Yoo SK, Cheong J, Kim HY. STAMPing into Mitochondria. Int J Biol Sci. 2014; 10(3):321-326.

https://doi.org/10.7150/ijbs.8456.

39. Wellen KE, Fucho R, Gregor MF, Furuhashi M, Morgan C, Lindstad T, et al. Coordinated regulation of nutrient and inflammatory responses by STAMP2 is essential for metabolic homeostasis. Cell. 2007;129(3):537-548.

https://doi.org/10.1016/j.cell.2007.02.049.

40. Fortier MA, Guilbault LA, Grasso F. Specific properties of epithelial and stromal cells from the endometrium of cows. J Reprod Fertil. 1988;83(1):239-248.

https://doi.org/10.1530/jrf.0.0830239.

41. Hailemariam D, Ibrahim S, Hoelker M, Drillich M, Heuwieser W, Looft C, et al. MicroRNA-regulated molecular mechanism underlying bovine subclinical endometritis. Reprod Fertil Dev. 2014;26(6):898913.

https://doi.org/10.1071/RD13027.

42. Jacca S, Franceschi V, Agosti M, Cavirani S, Mistretta F, Donofrio G. Interferon gamma-mediated BoHV-4 replication restriction in bovine endometrial stromal cells is host ID01 gene expression independent and BoHV-4 IE2 gene expression dependent. Biol Reprod. 2014; 91(5):112.

https://doi.org/10.1095/biolreprod.114.123000

43. Kanehisa M, Goto S, Kawashima S, Okuno Y, Hattori M. The KEGG resource for deciphering the genome. Nucleic Acids Res. 2004;32(Database issue):D277-280.

https://doi.org/10.1071/RD13027.

44. Kanehisa M, Furumichi M, Tanabe M, Sato Y, Morishima K. KEGG: new perspectives on genomes, pathways, diseases and drugs. Nucleic Acids Res. 2017; 45(D1):D353-d361.

https://doi.org/10.1093/nar/gkw1092.

45. Huang DW, Sherman BT, Lempicki RA. Bioinformatics enrichment tools: paths toward the comprehensive functional analysis of large gene lists. Nucleic Acids Res. 2009;37(1):1-13. 
https://doi.org/10.1093/nar/gkn923.

46. Pfaffl MW. A new mathematical model for relative quantification in real-time RT-PCR. Nucleic Acids Res. 2001;29(9):e45.

https://doi.org/10.1093/nar/29.9.e45.

\section{Tables}

Table 1 Data filtering statistics after Illumina sequencing

\begin{tabular}{lllllll}
\hline Sample & Raw Reads & Clean Reads No. & Clean Data (bp) & Clean Reads \% & Q20 (\%) & Q30 (\%) \\
& & & & & \\
\hline PBS1 & 47469396 & 44046138 & 6650966838 & 92.78 & 96.08 & 91.45 \\
PBS2 & 46293936 & 42854784 & 6471072384 & 92.57 & 96.49 & 92.24 \\
\hline PBS3 & 43441712 & 40242110 & 6076558610 & 92.63 & 96.25 & 91.79 \\
\hline LPS1 & 42929910 & 39806986 & 6010854886 & 92.72 & 96.19 & 91.59 \\
\hline LPS2 & 50813700 & 47094016 & 7111196416 & 92.67 & 96.16 & 91.6 \\
\hline LPS3 & 49082750 & 45600808 & 6885722008 & 92.9 & 96.42 & 91.9 \\
\hline
\end{tabular}

$\mathrm{N} \%$ : The percentage of fuzzy bases

Q20: The percentage of bases with a Phred value $>20$

Q30: The percentage of bases with a Phred value $>30$

Table 2 Summary of clean reads mapped to the reference genome

\begin{tabular}{|c|c|c|c|}
\hline $\begin{array}{ll}\text { e } & \text { Clean_Reads } \\
44046138\end{array}$ & $\begin{array}{l}\text { Total_Mapped } \\
41598276(94.44 \%)\end{array}$ & $\begin{array}{l}\text { Multiple_Mapped } \\
2394502(5.76 \%)\end{array}$ & $\begin{array}{l}\text { Uniquely_Mapped } \\
39203774 \text { (94.24\%) }\end{array}$ \\
\hline 42854784 & $39556838(92.30 \%)$ & $2206194(5.58 \%)$ & 37350644 (94.42\%) \\
\hline 40242110 & 37935794 (94.27\%) & 2240965 (5.91\%) & 35694829 (94.09\%) \\
\hline 39806986 & $37586818(94.42 \%)$ & $2233726(5.94 \%)$ & $35353092(94.06 \%)$ \\
\hline 47094016 & $44530730(94.56 \%)$ & $2676831(6.01 \%)$ & 41853899 (93.99\%) \\
\hline 45600808 & $43007562(94.31 \%)$ & $2461139(5.72 \%)$ & $40546423(94.28 \%)$ \\
\hline
\end{tabular}


Clean Reads: The total number of sequences used for alignment

Total Mapped: The total number of sequences identical to the reference genome in clean reads $\mathrm{B}$ percentage is total mapped / clean reads

Multiple Mapped: The total number of sequences aligned to multiple locations, percentage is multiple mapped / total mapped

Uniquely Mapped $\square$ The total number of sequences aligned to only one location, percentage is uniquely mapped / total mapped

\section{Additional Files}

Additional file 1: Figure S1. PCA analysis of the expressed transcripts. The X-axis is the first principal component, and the $\mathrm{Y}$-axis is the second principal component. Different shapes represent different samples, and different colors represent different groups. (A: control group; B: LPS-induced group)

Additional file 2: Table S1. DEGs in the bovine endometrial stromal cells were treated with LPS compared with PBS (control). The upregulated genes are shown in purple; the downregulated genes are shown in blue. Inf: infinite.

Additional file 3: Table S2. The significant GO terms of DEGs.

Additional file 4: Table S3. KEGG enrichment analysis of DEGs.

Additional file 5: Table S4. QRT-PCR primers used in the study.

\section{Figures}




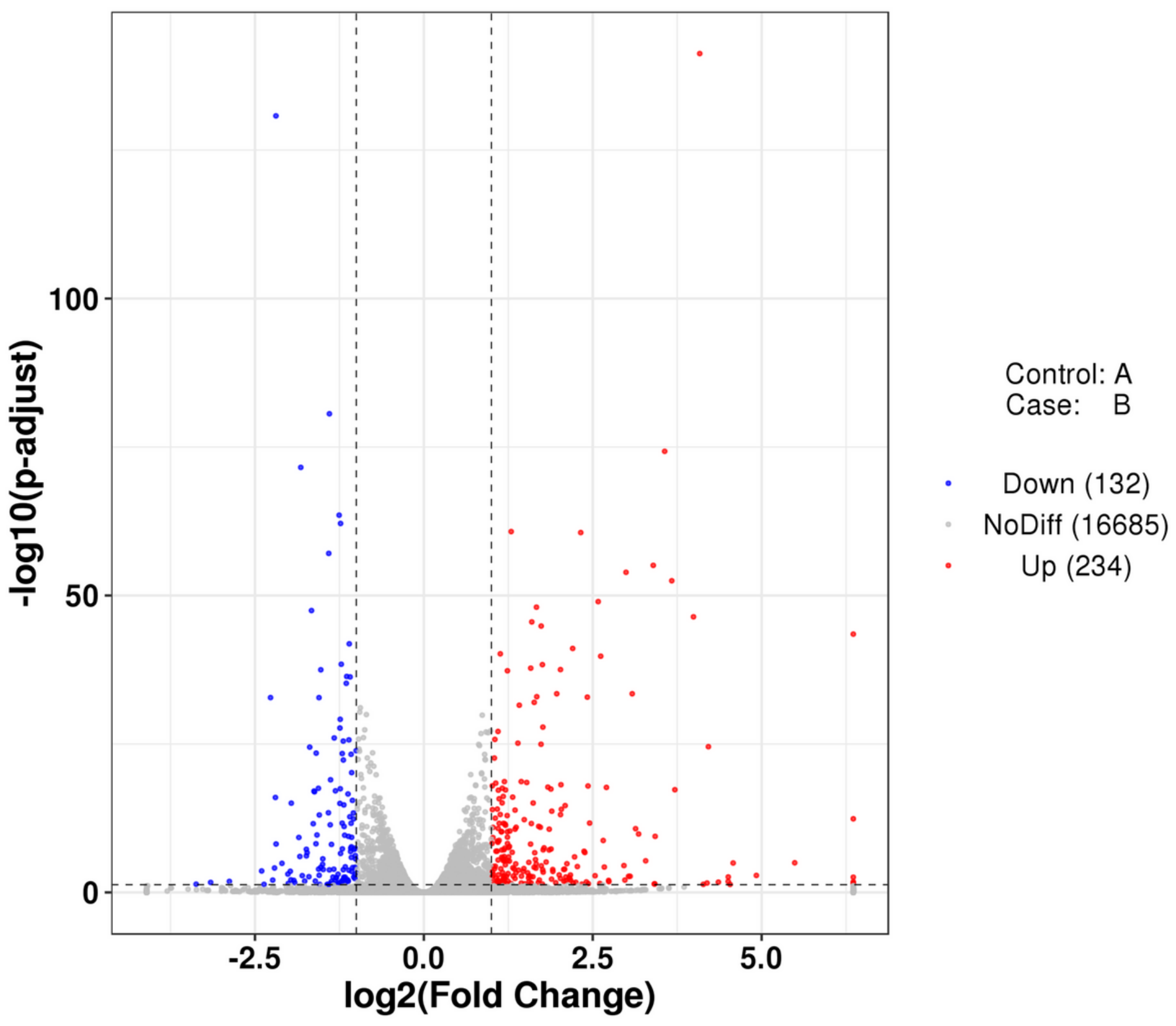

Figure 1

Volcano map of DEGs. The two vertical dotted lines are the threshold of differential expression. The horizontal dotted line is the threshold FDR of 0.05 . Upregulated and downregulated genes are shown as a red and blue dots, and gray dots represents non-significantly differentially expressed genes. (A: control group; B: LPS-induced group) 


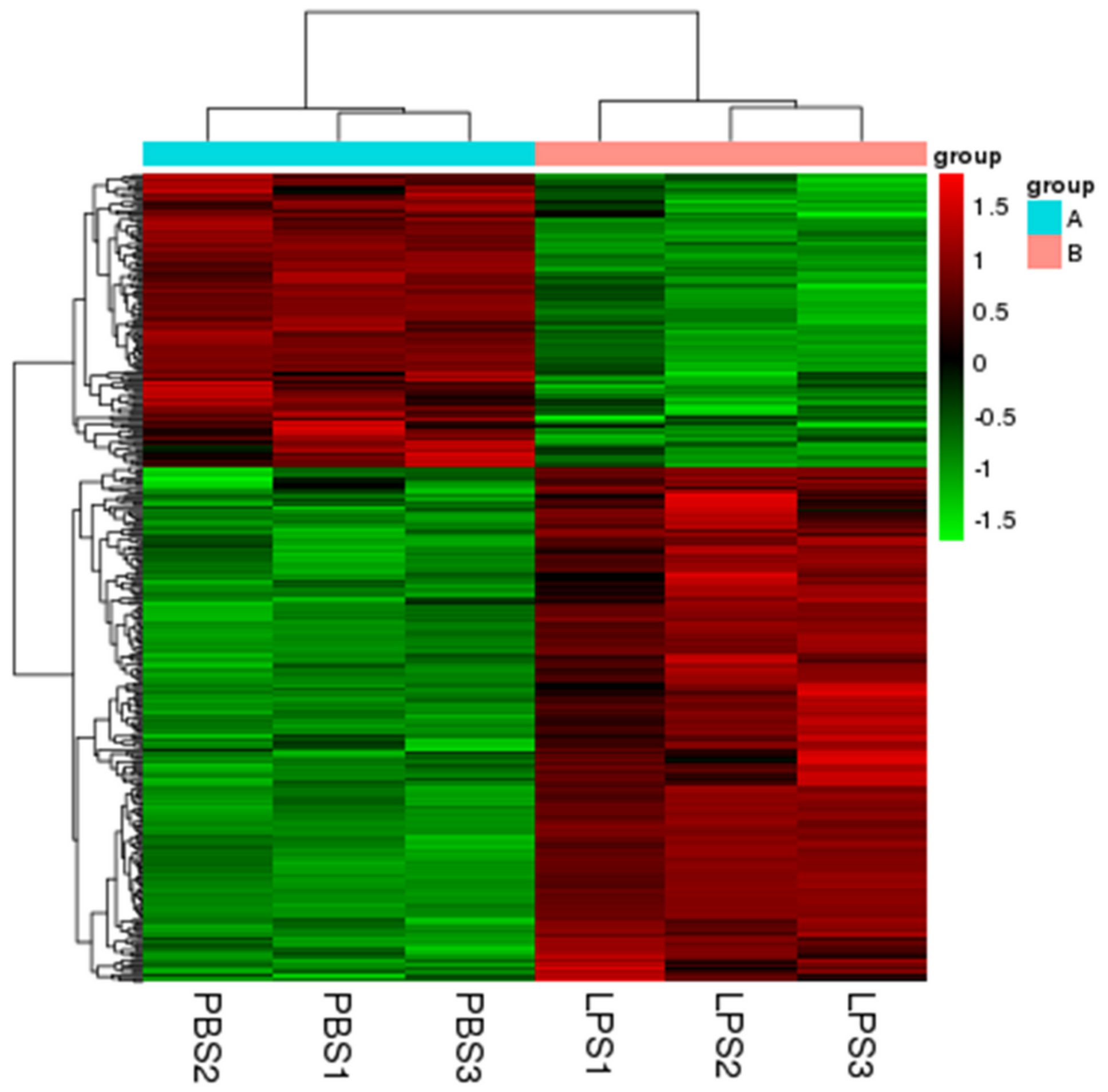

Figure 2

Heatmap analysis of DEGs. The horizontal lines represent genes, and each column is a sample. Red represents high-expression genes and green represents low-expression genes. The $\mathrm{X}$-axis is the sample number and the $\mathrm{Y}$-axis is the DEGs. 


\section{GO Enrichment}

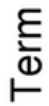

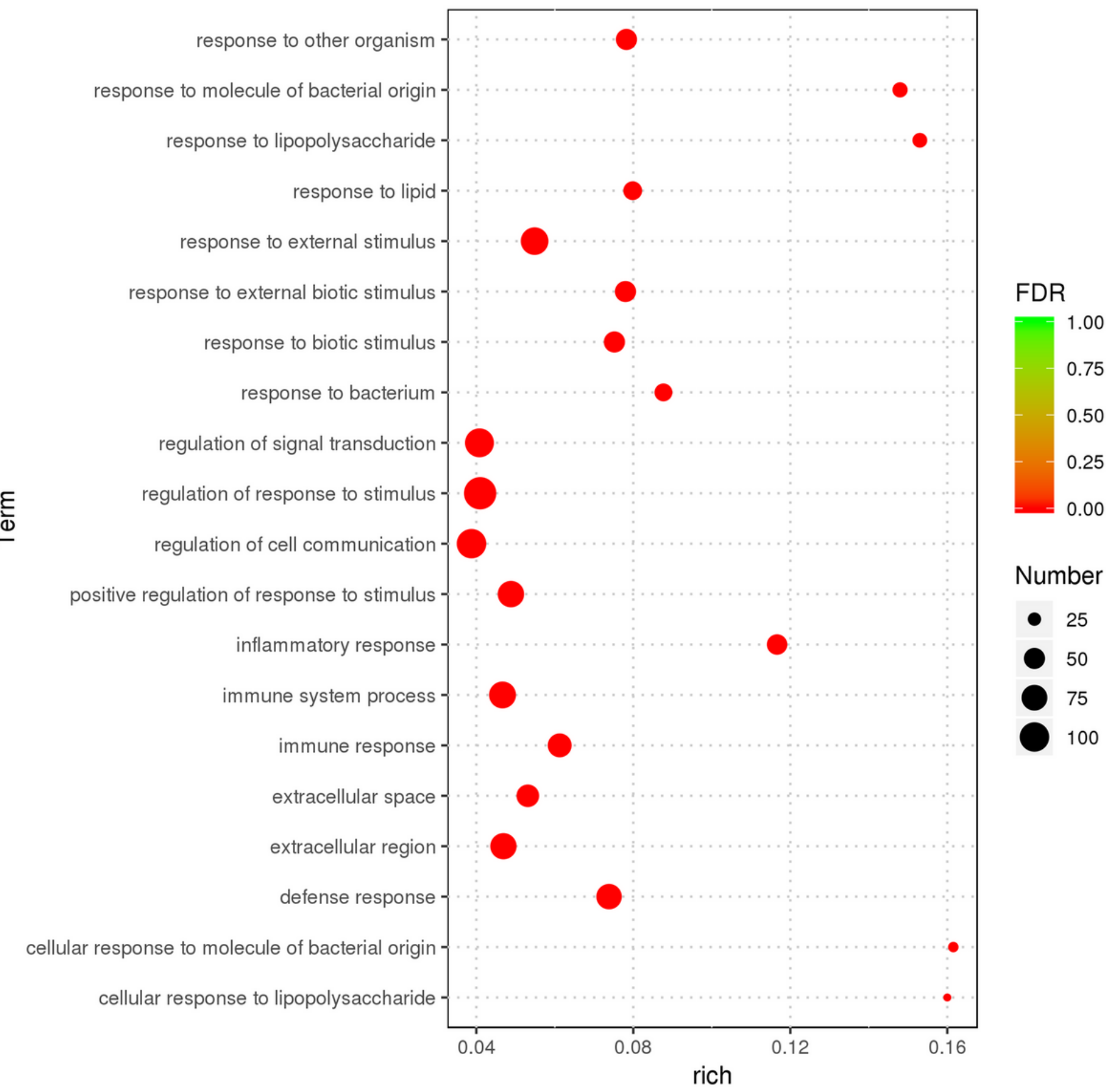

\section{Figure 3}

GO enrichment analysis displaying the first $20 \mathrm{GO}$ terms with the most significant enrichment. 


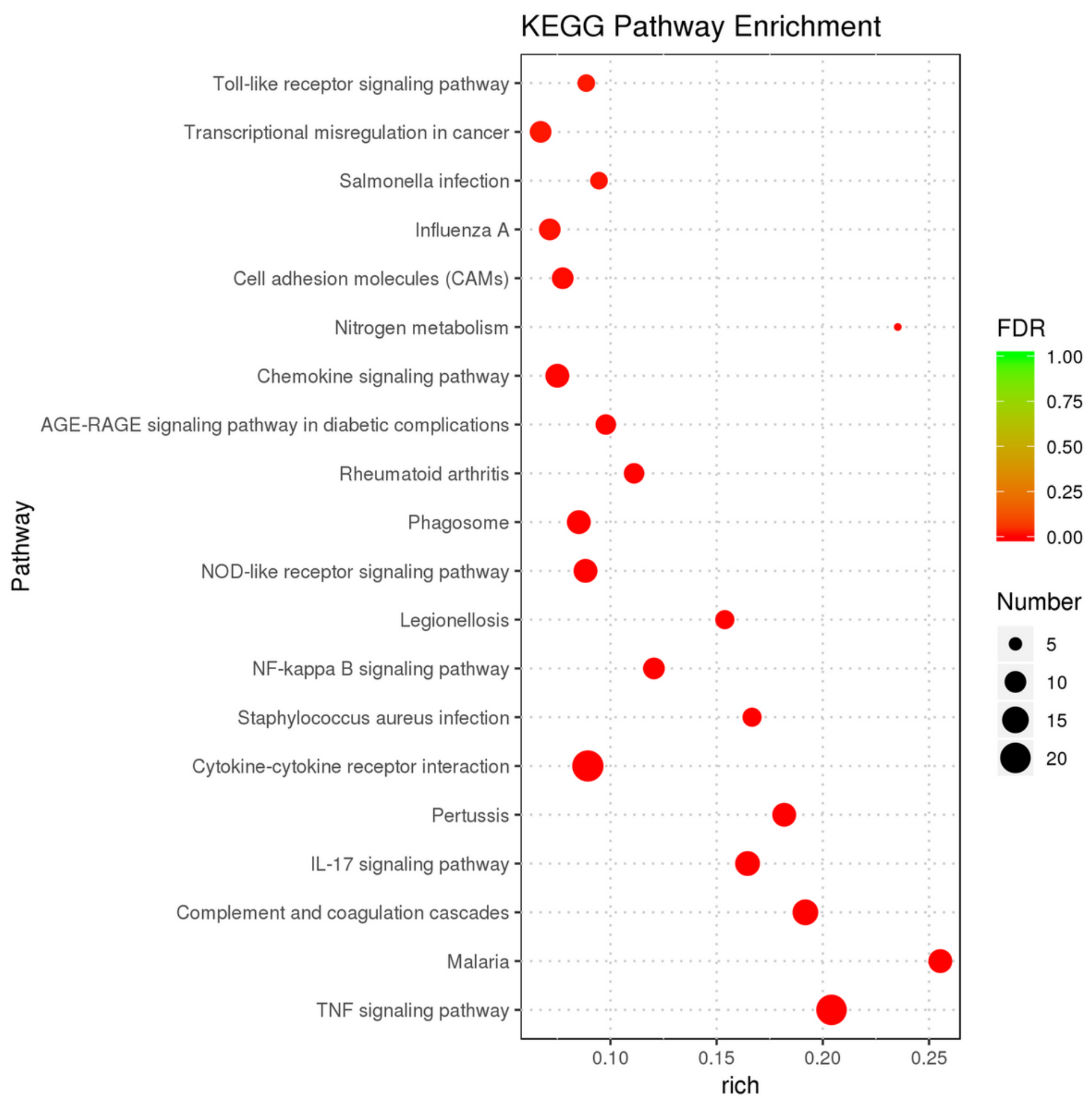

\section{Figure 4}

KEGG enrichment analysis displaying the first 20 KEGG terms with the most significant enrichment. 

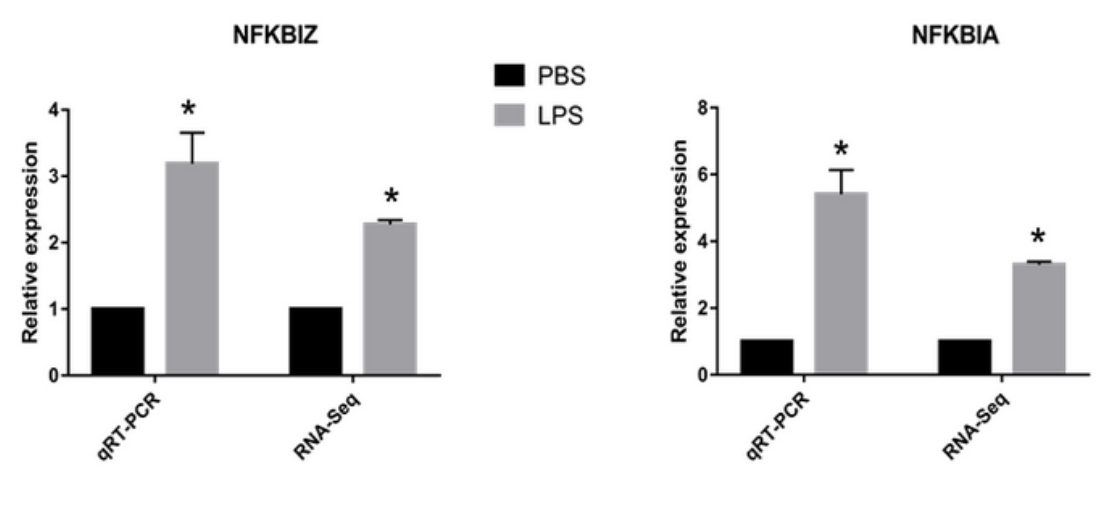

PBS
LPS

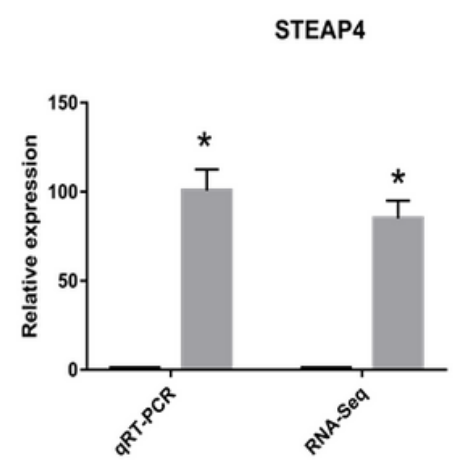

- PBS
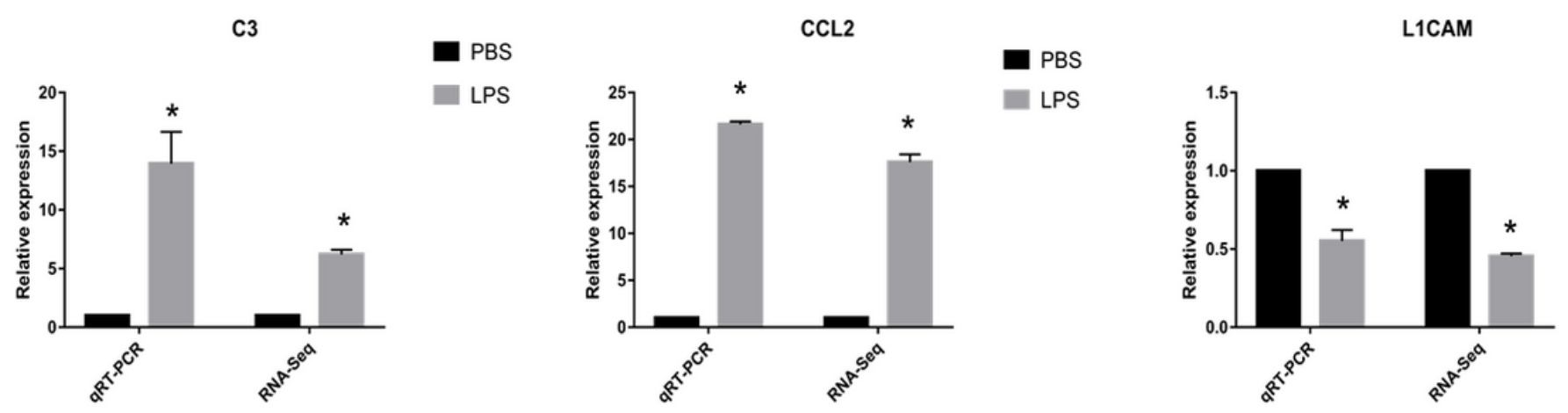

Figure 5

RT-PCR verified features of DEGs by RNA-seq. The relative expression level of target mRNAs was calculated using the 2- $\Delta \Delta \mathrm{Ct}$ method and expressed relative to the value in the control group. Results were displayed in mean \pm SEM $(n=3)$. Log2 fold change was the ratio of average log2 folds between groups. 


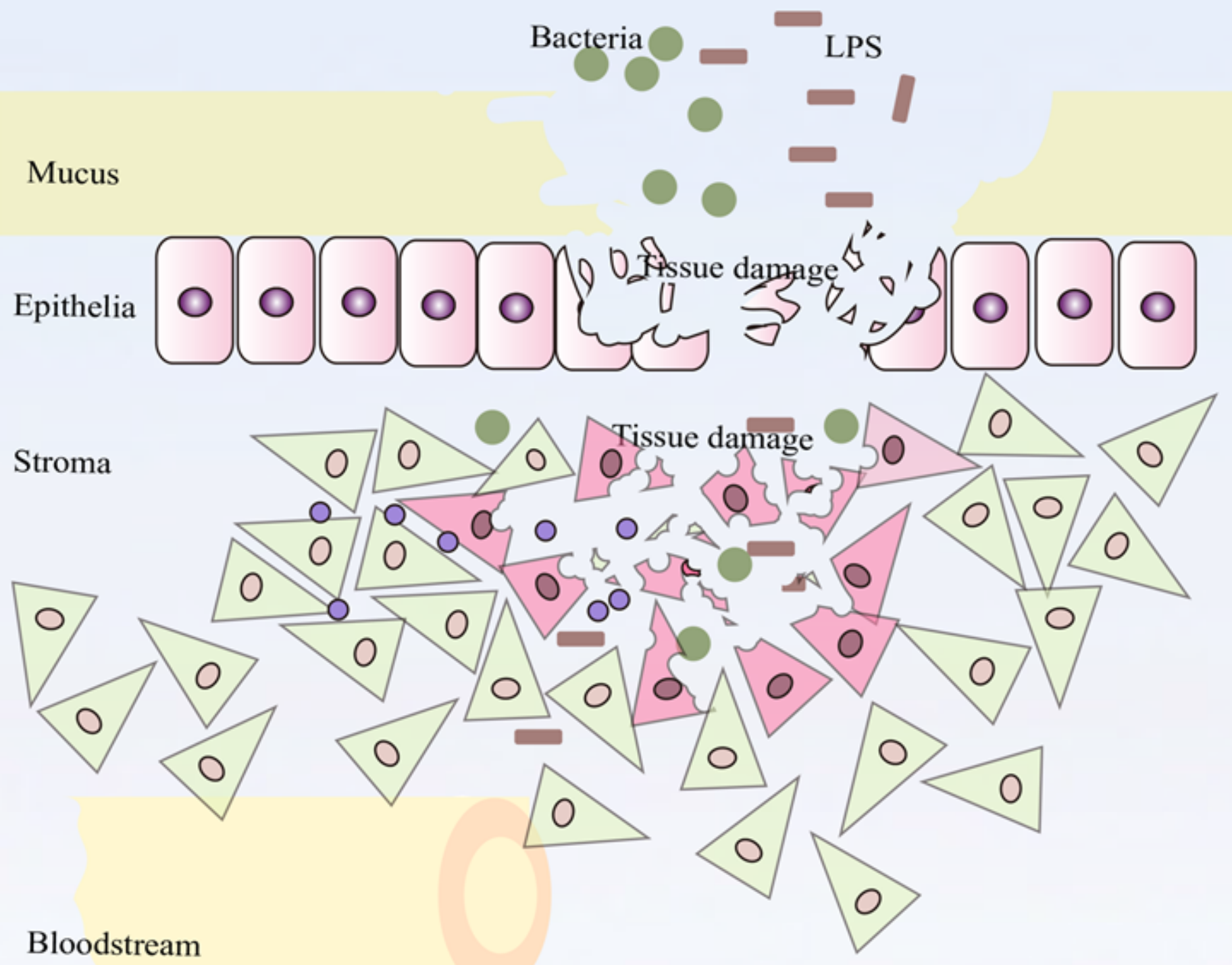

Figure 6

Stromal cells were exposed to an inflammatory environment when epithelial cells were disrupted. 


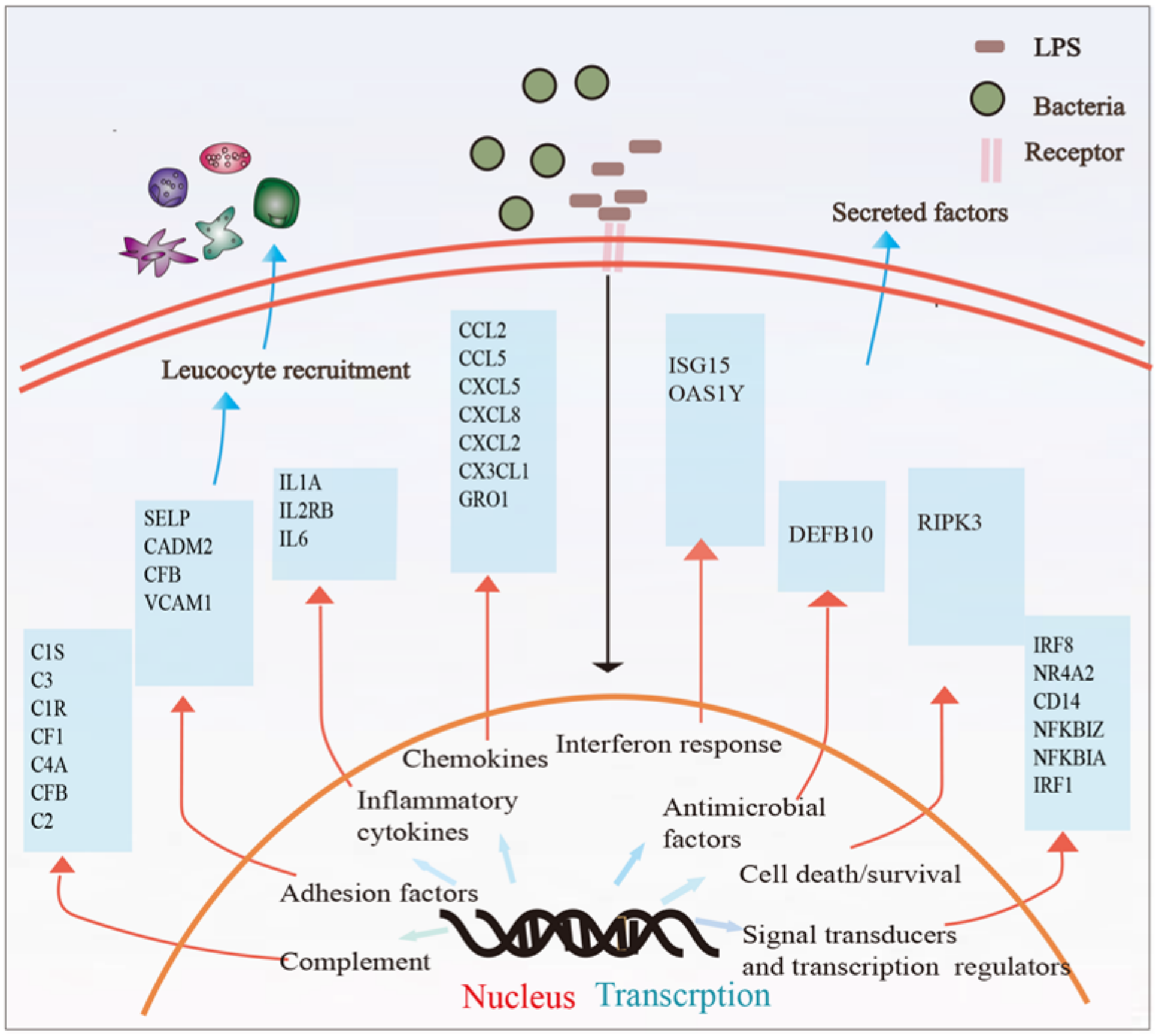

Figure 7

Summary of the immune response in bovine endometrial stromal cells exposed to bacterial LPS. LPS treatment for $12 \mathrm{~h}$ changed the mRNA expression of many genes involved inflammatory and innate immune response.

\section{Supplementary Files}

This is a list of supplementary files associated with this preprint. Click to download.

- Additionalfile1.tif 
- Additionalfile3.xIsx

- Additionalfile2.xIsx

- Additionalfile5.doc

- Additionalfile4.xlsx 